\title{
Negative Pressure Wound Therapy over the Achilles Tendon for Medically Compromised Ambulatory Patients
}

\author{
Woong Gyu Na, Hyoseob Lim, Sung Won Jung, Sung Hoon Koh \\ Department of Plastic and Reconstructive Surgery, Hallym University Sacred Heart Hospital, Hallym University Medical Center, Anyang, Korea
}

\begin{abstract}
Reconstruction of posterior ankle defects with Achilles tendon exposure caused by compromised medical conditions or trauma is a challenging issue. We present 2 cases which were successfully covered using negative pressure wound therapy (NPWT). In case 1, a 66-year-old man with a compromised status was referred for treatment of a diabetic ulcer on the right posterior ankle. Copious debridement resulted in Achilles tendon exposure. Eight weeks of NPWT followed by a split-thickness skin graft resulted in healing of the defect. In case 2, a 79-year-old male presented with a thermal burn from contact with a motorcycle muffler. Repetitive debridement exposed the Achilles tendon. Successful granulation tissue coverage was observed after 8 weeks of NPWT. There are many surgical methods to cover the exposed Achilles tendon including a local flap, free flap, or cross-leg flap, but these methods require some period of bed rest, which increases the risk of deep vein thrombosis, bedsores, and pneumonia. Bed rest also increases bone resorption and decreases bone formation, inducing osteoporosis and renal stones. However, healing by secondary intention using NPWT does not require any ambulation limitation and may be an effective reconstructive method in cardiovascularly compromised and elderly patients.
\end{abstract}

Keywords: Achilles tendon, Bed rest, Walking

\section{Introduction}

Posterior ankle defects that result in Achilles tendon exposure can either be caused by compromised medical conditions such as diabetes mellitus (DM) and peripheral vascular disease or by external trauma. These defects are challenging to reconstruct, and healing by secondary intention utilizing negative pressure wound therapy (NPWT) might be an effective treatment option. We present our experience with successful coverage of the exposed Achilles tendon in two patients using NPWT with active walking.

\section{Case 1}

A 66-year-old man with chronic kidney disease, chronic obstructive pulmonary disease, DM, and other comorbidities such as hypertension, atrial fibrillation, ischemic cardiomyopathy and periopehral arterial occlusion of lower extremities was referred for treatment of a diabetic ulcer on the right posterior ankle. Eschar was initially debrided, resulting in a defect with remnant non-demarcated necrotic tissue (Fig. 1A). NPWT using CuraVAC ${ }^{\circledR}$ (CGBio, Seongnam, Korea) was employed to stimulate rapid growth of granulation tissue. CuraVAC ${ }^{\circledR}$ was changed every 3 days in the operative field and kept with $80 \mathrm{mmHg}$ to $120 \mathrm{mmHg}$ cyclic mode. Inner unhealthy granulation tissue and local debris covering the Achilles tendon were removed while changing the apparatus, which resulted in a $3.0 \mathrm{~cm} \times 1.8 \mathrm{~cm}$ sized area of tendon expo-

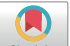 \\ Case Report \\ Received: May 30, 2017 \\ Revised: August 22, 2017 \\ Accepted: August 25, 2017 \\ Corresponding author: \\ Hyoseob Lim, M.D., Ph.D \\ Department of Plastic and Reconstructive \\ Surgery, Hallym University Sacred Heart \\ Hospital, Hallym University Medical Center, \\ 22 Gwanpyeong-ro 170beon-gil, Dongan-gu, \\ Anyang 14068, Korea \\ Tel: +82-31-380-3781 \\ Fax: +82-31-380-5980 \\ E-mail: hyoseob.lim@gmail.com \\ No potential conflict of interest relevant to this \\ article was reported. \\ This is an Open Access article distributed under the terms \\ of the Creative Commons Attribution Non-Commercia \\ which permits unrestricted non-commercial use, \\ distribution, and reproduction in any medium, provided the \\ original work is properly cited. \\ C 2017 Korean Wound Management Society
}



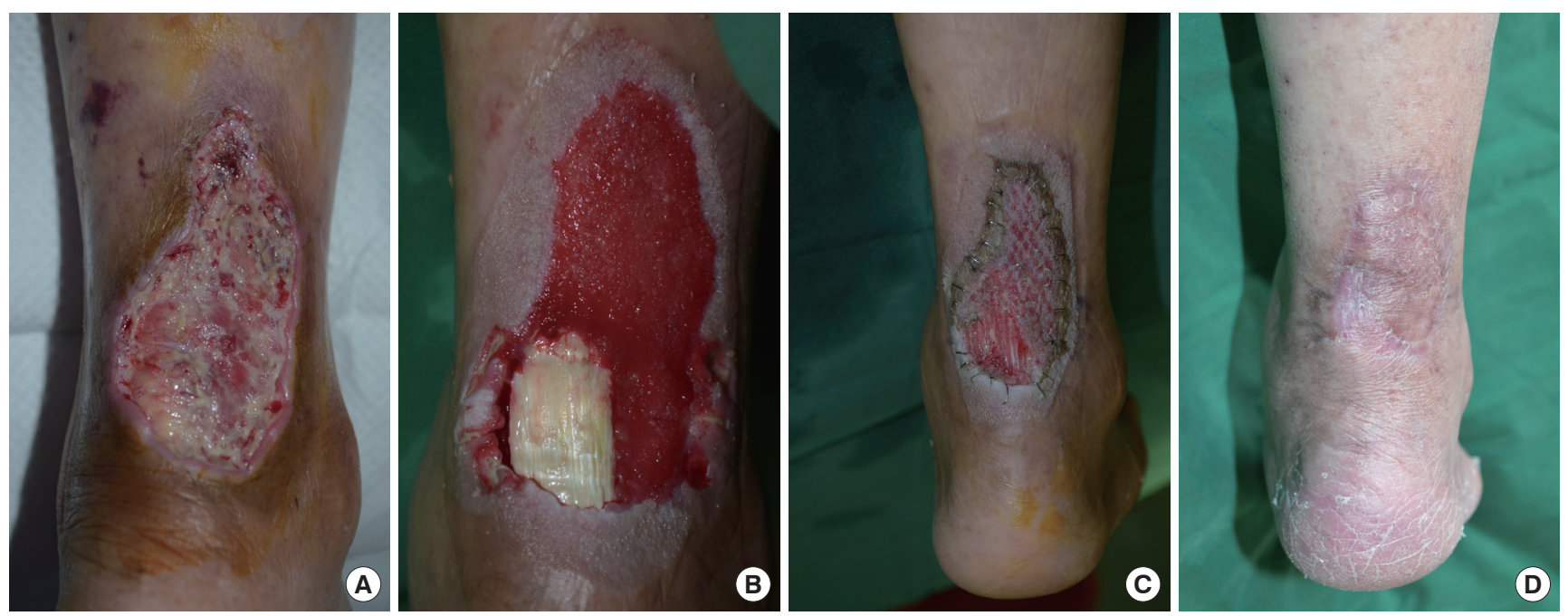

Fig. 1. A case of undemarcated diabetic ulcer on right posterior ankle. (A) Immediate photograph after initial escharectomy. (B) Photograph after 4 weeks of NPWT. (C) Postoperative 9 days photograph after STSG coverage. (D) Postoperative 11 months photograph after STSG coverage.

sure (Fig. 1B). The superficial paratendon was destroyed. Considering the patient's age and poor vascular status of lower extremities with intermittent claudication and blue toes, healing by secondary intention with NPWT rather than other operative management was employed, and we actively encouraged ambulation. Eight weeks after NPWT was started, the size of the exposed tendon area had diminished, and the surrounding soft tissue defect was filled with healthy granulation tissue. A split-thickness skin graft with 1:1.5 mesh expansion was placed on the surrounding defects and the exposed tendon 62 days after NPWT was started. NPWT was continuously applied to the recipient site for skin fixation with $120 \mathrm{mmHg}$ continuous mode. After 1 week, the grafted skin over the exposed tendon had not taken. However, granulation tissue covered the exposed tendon using the split skin as a biologic dressing, while successful grafting took place in an adjacent area (Fig. 1C). Twenty-eight days postoperatively, the patient was discharged with a small remaining posterior ankle defect. The patient changed the foam or gauze dressing materials daily, and complete epithelization was observed on outpatient follow-up 48 days postoperatively. Eleven months later, complications such as limited motion range, hypertrophic scarring, and wound dehiscence had not occurred (Fig. 1D).

\section{Case 2}

A 79-year-old male presented to our clinic for treatment of a thermal burn in the right calf and posterior ankle area (3.5\% of the total body surface area) caused by contact with a motorcycle muffler. Upon admission, the posterior ankle area was covered with necrotic tissue (Fig. 2A). Repetitive debridement was done, and the Achilles tendon with a partially destroyed paratendon was exposed. Considering his advanced age and poor vascularity with adjacent tissue destruction caused by diffuse burn, healing by secondary intention using NPWT was planned. CuraVAC ${ }^{\circledR}$ was applied on the exposed paratendon with $80 \mathrm{mmHg}$ to $120 \mathrm{mmHg}$ cyclic mode and changed every 3 days. When changing the apparatus, the damaged paratendon was meticulously debrided, and several longitudinal incisions that were expected to promote healing were made in the tendon (Fig. 2B). These longitudinal slits penetrated the Achilles tendon from superficial surface to inner surface and were expected to promote vascular growth from posterior vessels and were made in multiple sites using the number 11 blade. After 29 days of continuous NPWT, the exposed tendon was covered with granulation tissue (Fig. 2C). For the purpose of full mobilization before grafting, ambulation was encouraged during the period of NPWT application, and the patient was discharged and recommended to continue ambulation for about 2 weeks. Thirteen days later, a split-thickness skin graft was performed using 1:1.5 mesh expansion, and $\mathrm{CuraVAC}^{\circledR}$ with $120 \mathrm{mmHg}$ continuous mode was used to fixed the skin. The meshed skin was successfully taken on the surrounding defect but failed to cover the Achilles area because of the mobile tendon 

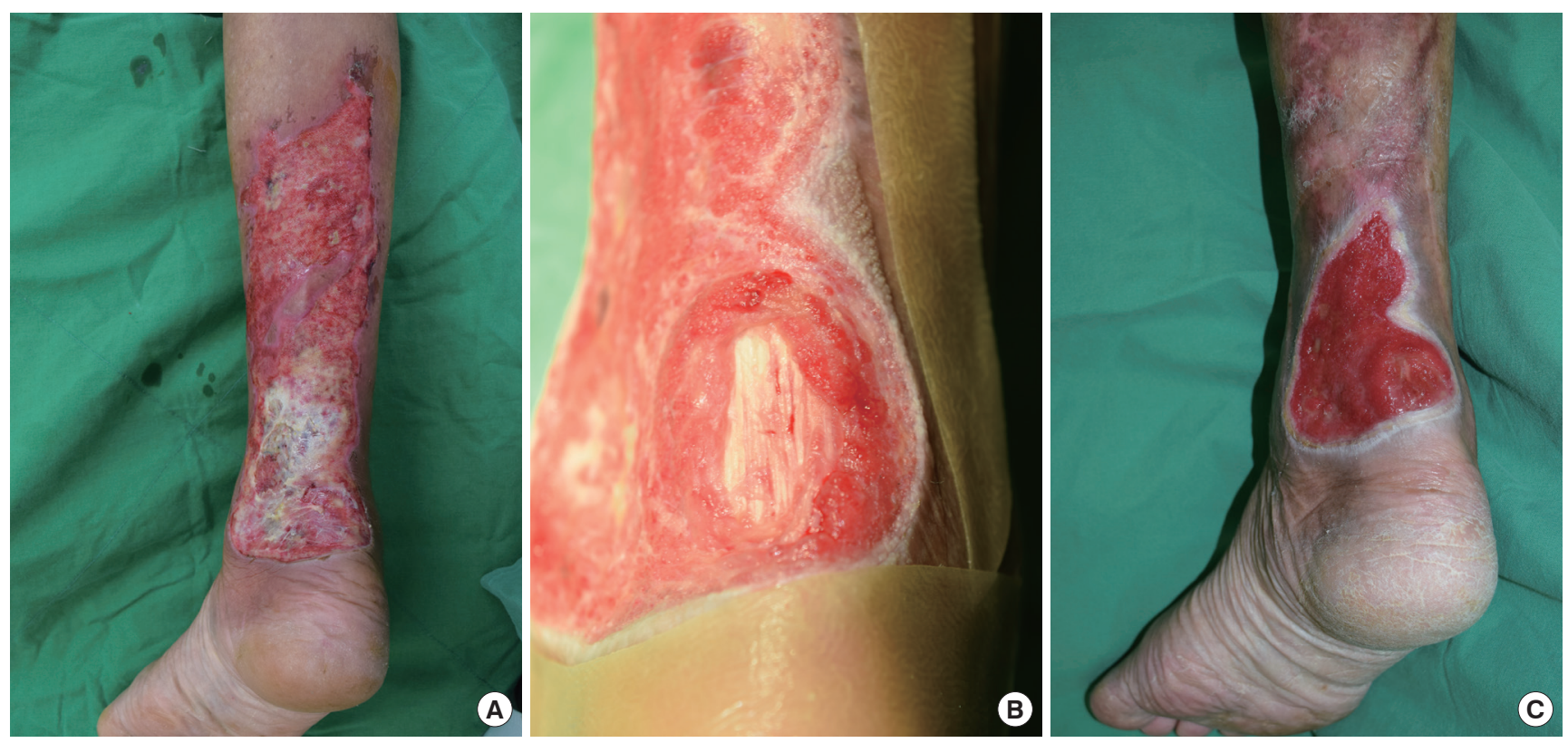

Fig. 2. A case of contact thermal burns on right calf and ankle. (A) Initial photograph when the patient admitted. (B) Photograph showing longitudinal incision on Achilles tendon. (C) Photograph after 29 days of NPWT.

and overlying granulation tissue. He was discharged with a $2.0 \mathrm{~cm} \times 2.3 \mathrm{~cm}$ sized area of raw surface without tendon exposure. The Achilles tendon area finally healed after self-application of foam and gauze dressing.

\section{Discussion}

There are many surgical methods to cover an exposed Achilles tendon. Local flaps which utilize healthy soft tissue are not appropriate if the adjacent soft tissue is injured [1,2]. Microsurgical free tissue transfers and cross-leg flaps are other options for lower extremity reconstruction [1,2]. However, free flaps may be not good choices when adequate recipient vessels are absent [3]. Endovascular angioplasty combined with free soft tissue transfer has been common over the past decade for limb salvage but has a high restenosis rate and is relatively contraindicated in patients with severe chronic renal insufficiency $[4,5]$. A cross-leg flap requires prolonged immobilization, longer hospitalization, and additional procedures for flap division [6].

NPWT has been used previously for open wound management [7]. Several healing mechanisms, including pressureinduced wound size reduction called macrodeformation, occur in NPWT. Multiple cytokines are involved in healing at the molecular level. In the literature, systemic IL-10 and local IL-8 were reported to be increased [8]. In our cases and other lit- erature, exposed Achilles tendons even with superficial paratendon destruction were successfully covered with granulation tissue using NPWT $[1,2,9]$. Various methods were used in our cases. In case 1 , split-thickness skin was used as a biologic dressing to enhance granulation tissue growth. In the literature, honey, cultured epithelial autograft, human amnion, and other commercial allograft products have been used for biologic treatment [10]. A failed flap was used to cover a scalp defect, resulting in successful granulation tissue growth [11]. In case 2, longitudinal slits penetrating the Achilles tendon were made to promote rapid healing, which has been reported to be effective by Erika Ohata et al [1]. Incisions penetrating the Achilles tendon may induce posterior blood flow to the superficial surface and facilitate granulation tissue growth over the tendon with NPWT [1].

The time period of NPWT required for covering the defect varies and might be affected by underlying conditions such as age, DM, and peripheral vascular disease. Our cases took about 8 weeks and 4 weeks, respectively, to cover the defect with granulation tissue even with active exercise and walking that may have induced device nonadherence. Repta et al. reported NPWT over the Achilles tendon in 3 vascularly compromised patients who required 7,4 , and 12 weeks, respectively, for the complete healing by secondary intention, but Heugel et al. reported that healing took place in only 12 days in a 16-year-old female without any underlying disease [2,9]. 
Other surgical methods require a period of bed rest, but bed rest increases bone resorption and decreases bone formation, inducing osteoporosis and renal stones [12]. Bed rest also increases the risk of deep vein thrombosis, bedsores, and pneumonia, which are critical conditions for elderlyindividuals [13]. However, with NPWT, active ambulation is possible. Our patients are encouraged to ambulate with the VAC apparatus even after skin grafting.

In conclusion, NPWT may be an effective method to cover the exposed Achilles tendon in cardiovascularly compromised and elderly patients because active ambulation is possible.

\section{References}

1. Ohata E, Yuzuriha S, Mishima Y, et al. Longitudinal slit procedure in addition to negative pressure wound therapy for a refractory wound with exposed achilles tendon. Eplasty 2015 18;15:e9. eCollection 2015.

2. Heugel JR, Parks KS, Christie SS, et al. Treatment of the exposed Achilles tendon using negative pressure wound therapy: a case report. J Burn Care Rehabil 2002;23:167-71.

3. Hung SJ, Chen HC, Wei FC. Free flaps for reconstruction of the lower back and sacral area. Microsurgery 2000;20:72-6.

4. Chou C, Kuo PJ, Chen YC, et al. Combination of Vascular Intervention Surgery and Free Tissue Transfer for Critical Diabet- ic Limb Salvage. Ann Plast Surg 2016;77 Suppl 1: S16-21.

5. Lucas LC, Mills JL Sr. Critical evaluation of endovascular surgery for limb salvage. Plast Reconstr Surg 2011;127 Suppl 1:163S-173S.

6. Lineaweaver W, Zhang F. Cross-leg flaps and reconstructive surgery in the 21st century. Ann Plast Surg 2014;72:491-2.

7. Huang $C$, Leavitt $T$, Bayer LR, et al. Effect of negative pressure wound therapy on wound healing. Curr Probl Surg 2014; 51:301-31.

8. Glass GE, Murphy GF, Esmaeili A, et al. Systematic review of molecular mechanism of action of negative-pressure wound therapy. Br J Surg 2014;101:1627-36.

9. Repta R, Ford R, Hoberman L, et al. The use of negativepressure therapy and skin grafting in the treatment of softtissue defects over the Achilles tendon. Ann Plast Surg 2005; 55:367-70.

10. Lineen E, Namias N. Biologic dressing in burns. J Craniofac Surg 2008;19:923-8.

11. Chadwick S, Kosutic D. The use of a failed flap as a biological dressing. Ann R Coll Surg Engl 2016;98:e19-21.

12. Okada A, Ohshima H, Itoh Y, et al. Risk of renal stone formation induced by long-term bed rest could be decreased by premedication with bisphosphonate and increased by resistive exercise. Int J Urol 2008;15:630-5.

13. Allen C, Glasziou P, Del Mar C. Bed rest: a potentially harmful treatment needing more careful evaluation. Lancet 1999; 9:354:1229-33. 